\title{
Epigenetic Regulation by Sulforaphane: Opportunities for Breast and Prostate Cancer Chemoprevention
}

\author{
Lauren L. Atwell • Laura M. Beaver • Jackilen Shannon • \\ David E. Williams • Roderick H. Dashwood • Emily Ho
}

Published online: 30 January 2015

(C) Springer International Publishing AG 2015

\begin{abstract}
Sulforaphane (SFN) is a phytochemical derived from cruciferous vegetables that has multiple molecular targets and anti-cancer properties. Researchers have demonstrated several chemopreventive benefits of SFN consumption, such as reductions in tumor growth, increases in cancer cell apoptosis, and disruption of signaling within tumor microenvironments both in vitro and in vivo. Emerging evidence indicates that SFN exerts several of its chemopreventive effects by altering epigenetic mechanisms. This review summarizes evidence of the impact of SFN on epigenetic events and how they relate to the chemopreventive effects of SFN observed in preclinical and clinical studies of breast and prostate cancers. Specific areas of focus include the role of SFN in the regulation of cell cycle, apoptosis, inflammation, antioxidant defense, and cancer cell signaling and their relationships to epigenetic mechanisms. Finally, remaining challenges and research needs for translating mechanistic
\end{abstract}

This article is part of the Topical Collection on Epigenetics and Phytochemicals

L. L. Atwell · L. M. Beaver • E. Ho

School of Biological and Population Health Sciences, Oregon State University, 103 Milam Hall, Corvallis, OR 97331, USA

\section{L. Atwell}

e-mail: atwell1@onid.oregonstate.edu

\section{M. Beaver}

e-mail: Laura.Beaver@oregonstate.edu

L. M. Beaver • D. E. Williams $\cdot$ E. Ho

Linus Pauling Institute, Oregon State University, 307 Linus Pauling

Science Center, Corvallis, OR 97331, USA

D. E. Williams

e-mail: david.williams@oregonstate.edu

\section{J. Shannon}

Department of Public Health and Preventive Medicine, Oregon Health and Science University, 3181 SW Sam Jackson Park Road,

Mail Code CB L606, Portland, OR 97239, USA work with SFN into human studies and clinical intervention trials are discussed.

Keywords Sulforaphane $\cdot$ Cancer $\cdot$ Chemoprevention $\cdot$ Epigenetics $\cdot$ Breast $\cdot$ Prostate

\section{Introduction}

Epidemiological evidence suggests that consuming cruciferous vegetables $(\mathrm{CV})$, such as broccoli and cauliflower, may lower risks of developing breast and prostate cancers [1]. Sulforaphane (SFN), a phytochemical derived from these vegetables, possesses many of the chemopreventive properties associated with consuming CV [2]. SFN is produced from glucoraphanin (GFN), a glucosinolate precursor found in CV.

\section{E. Williams}

Department of Environmental and Molecular Toxicology, Oregon State University, 1007 Agriculture \& Life Sciences Building, Corvallis, OR 97331, USA

\section{R. H. Dashwood}

Center for Epigenetics \& Disease Prevention, 2121 West Holcombe Boulevard, Houston, TX 77030, USA

e-mail: rdashwood@ibt.tamhsc.edu

\section{E. Ho $(\square)$}

Moore Family Center for Whole Grain Foods, Nutrition and Preventive Health, Oregon State University, 212 Milam Hall, Corvallis, OR 97331, USA

e-mail: Emily.Ho@oregonstate.edu 
GFN is converted to SFN via the plant enzyme myrosinase. SFN is metabolized via the mercapturic acid pathway, generating several bioactive metabolites [3]. Early research has focused on the ability of SFN to activate nuclear factor (erythroid-derived 2)-like 2 (Nrf2) and induce phase 2 enzymes, as well as inhibit enzymes involved in activating carcinogens [4]. However, there has been growing interest in alternative mechanisms of chemoprevention by SFN that include epigenetic targets [5]. Epigenetics refers to heritable changes in gene expression that are not caused by changes in DNA sequence. Epigenetic mechanisms work in concert to produce changes in chromatin structure and gene expression by modifying interactions among DNA, transcriptional machinery, and regulatory protein complexes. Major epigenetic mechanisms include histone modifications, DNA methylation, noncoding RNAs (ncRNAs), and chromatin remodeling. Unlike genetic mutations, epigenetic alterations are potentially reversible, making them attractive targets for cancer chemoprevention [6].

Dysregulation of epigenetic mechanisms is emerging as an important factor in cancer development and progression. Epigenetic alterations that improperly silence tumor suppressor genes and activate oncogenes allow cells to acquire cancerpromoting properties, such as uninhibited cell growth and proliferation [7]. In breast and prostate cancers, alterations in the expression of histone deacetylases (HDACs), histone methyltransferases (HMTs), and miRNAs, as well as altered levels of histone modifications and DNA methylation, have been observed [8-11]. Reversing these aberrant epigenetic alterations is becoming a focus of many chemopreventive strategies. This review summarizes evidence from preclinical and clinical studies, with a focus on work conducted in vivo, that demonstrates the ability of SFN to attenuate breast and prostate carcinogenesis through epigenetic mechanisms (Fig. 1). Considerations for translating mechanistic work with SFN into human studies are also discussed.

\section{SFN and Epigenetics in Prostate and Breast Cancer}

SFN has been shown to alter key epigenetic mechanisms in vivo and in vitro with corresponding impact on prostate and breast cancer development. Histone modifications, which occur on histone tails, alter interactions between histones and DNA and affect gene transcription. Histone acetyltransferases (HATs) add acetyl groups to lysine residues within histone tails, thereby relaxing the chromatin structure and facilitating activation of gene transcription. Histone deacetylases (HDACs) suppress gene transcription by removing these acetyl groups [6]. Reduced HDAC activity in peripheral blood mononuclear cells (PBMCs), prostates, and prostate cancer cell xenografts were reported in mice that consumed $443 \mathrm{mg} / \mathrm{kg}$ of SFN in the diet for 3 weeks [12]. Prostates and xenografts exhibited corresponding increases in global acetylation of histones $\mathrm{H} 3$ and H4. Importantly, site-specific increases in histone acetyl marks were observed at gene promoters for $\mathrm{p} 21$ and Bax in tissues with corresponding increases in gene expression [13]. Additionally, SFN reduced protein levels of specific HDACs in prostate and breast cancer cell lines at concentrations ranging from 1 to $15 \mu \mathrm{M}[14,15 \bullet$,

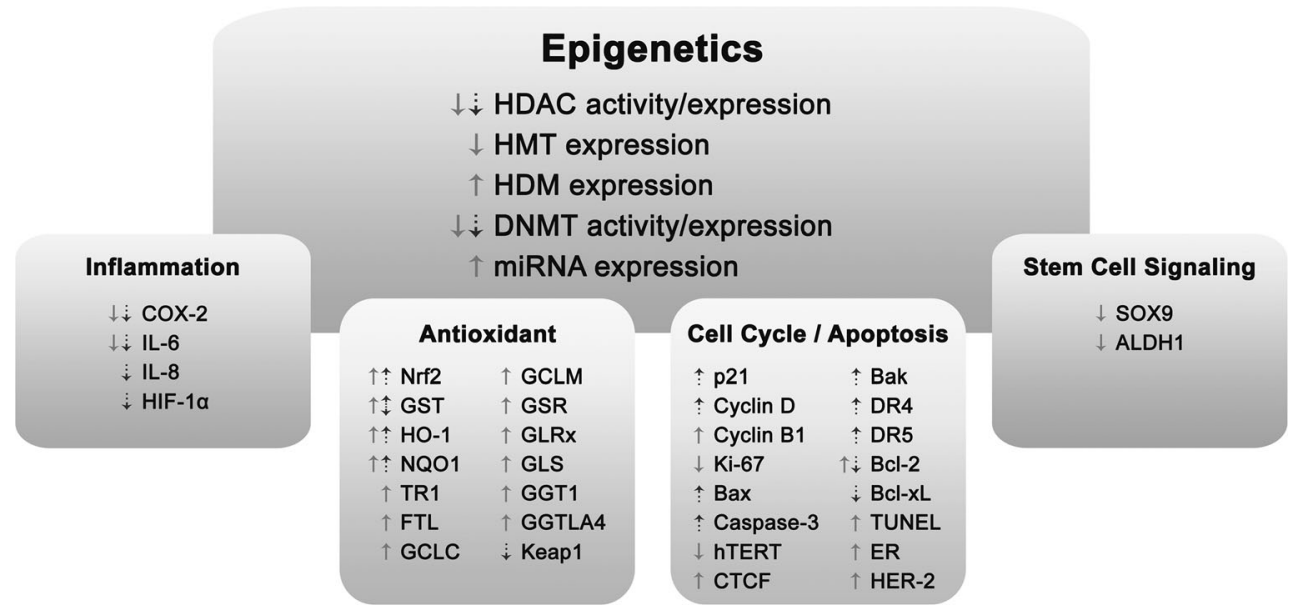

Fig. 1 SFN-induced changes in epigenetic-modifying enzymes and SFN targets that may be regulated in part through epigenetic mechanisms. Pictured are multiple targets relevant to breast (solid arrows) and prostate (dotted arrows) cancer prevention. ALDH1 aldehyde dehydrogenase 1 family, member A1, CTCF CCCTC-binding factor, $C O X-2$ cyclooxygenase-2, DNMT DNA methyltransferases, $D R$ death receptor, ER estrogen receptor, FTL ferritin, light polypeptide, GCLC glutamate-cysteine ligase, catalytic unit, GCLM glutamate-cysteine ligase, modifier unit, GGT1 gamma-glutamyltransferase 1, GGTLA4 gamma-glutamyltransferase-like activity 4, GLRx glutaredoxin 1, GLS glutaminase, GSR glutathione reductase, GST glutathione S-transferase, $H D A C$ histone deacetylase, HDM histone demethylase, HER-2 human epidermal growth factor receptor $2, H I F-1 \alpha$ hypoxia-inducible factor $1 \alpha, H M T$ histone methyltransferase, HO-1 heme oxygenase-1, hTERT human telomerase reverse transcriptase, IL interleukin, Keapl kelchlike ECH-associated protein 1, miRNA microRNA, NQO1 NAD(P)H: quinone oxidoreductase 1, Nrf2 nuclear factor (erythroid-derived 2)-like 2, SOX9 SRY (sex determining region Y)-box 9, TR1 thioredoxin reductase 1, TUNEL terminal nucleotidyl transferase-mediated nick end labeling. (Figure produced using Adobe Photoshop) 
16]. These concentrations have also been shown to inhibit HDAC activity and alter histone acetyl marks in breast cancer cell lines [16-18]. While SFN's effect on HAT expression and activity has received less attention, some studies have reported no change in HAT activity in breast cancer cell lines following SFN treatment $[16,18]$.

Emerging evidence suggests that SFN may alter additional epigenetic processes in the breast and prostate including DNA and histone methylation as well as ncRNAs. DNA methyltransferases (DNMTs) add methyl groups to cytosine bases in DNA. High levels of DNA methylation are generally associated with gene silencing. DNMT1, often referred to as the "maintenance" DNMT, maintains methylation patterns through cell division. In contrast, DNMT3a and DNMT3b are responsible for de novo methylation and methylate DNA during development and according to environmental signals [9]. In human and mouse breast and prostate cancer cell lines, SFN treatment decreased DNMT activity and protein levels of DNMT1 and DNMT3a at SFN concentrations ranging from 1 to $30 \mu \mathrm{M}$. As a consequence, attenuated global and sitespecific DNA methylation were linked to altered gene expression $[15 \bullet, 16,18-20]$. Histone methyltransferases (HMTs) add methyl groups generally to lysine and arginine residues, and histone demethylases (HDM) remove them. Changes in chromatin structure resulting from histone methylation depend on the number of methyl groups and the residue modified [9]. ncRNAs are produced from non-coding regions of DNA and also play critical roles in modifying the epigenome. miRNAs are ncRNAs that bind mRNAs with appropriate "seed sequences," which prevent the mRNA from being translated or enhance degradation of the mRNA template and have been implicated in cancer development [21, 22]. In human breast cancer cell lines, SFN treatment decreased protein levels of SUV39H1 (a HMT) and histone methyl marks (H3K27me3 and H3K9me3), increased protein levels of RBP2 (a HDM), and altered expression of the miRNA, miR140 , and its downstream targets [16, 18, 23•]. To our knowledge, a direct interaction between SFN and chromatin remodeling complexes (e.g., SWItch/Sucrose NonFermentable (SWI/SNF)) has not been established, but epigenetic events such as histone acetylation have been shown to influence nucleosome structure (reviewed by [24]).

\section{Prostate Cancer}

Chemopreventive properties of SFN have been demonstrated in the prostate in vivo. In mouse models of prostate cancer, SFN ingestion decreased tumor growth, increased cancer cell apoptosis, and prevented cancer progression $[25,26]$. Several groups reported that SFN reached prostate tissue in rodents following oral consumption and had localized effects [12, 13, $27 \bullet \bullet, 28]$. Much of the epidemiological evidence suggests that individuals who consume greater amounts of CV have a decreased risk of developing prostate cancer [29]. Additionally, a prospective study reported a decreased risk of prostate cancer progression with higher intakes of cruciferous vegetables [30]. There have also been a few human clinical prostate cancer trials conducted using SFN. In one such study involving men with recurrent prostate cancer, none of the patients experienced PSA doubling, a marker of disease severity, after consuming SFN daily for $<20$ weeks [31]. In men diagnosed with high-grade prostatic intraepithelial neoplasia, a 12-month dietary broccoli intervention altered gene expression in prostate tissues relating to cell signaling pathways such as androgen, TGF $\beta 1$, insulin, and EGF signaling [32]. These findings suggest a potential role of SFN in preventing prostate cancer. Emerging evidence suggests that SFN's chemopreventive activities in the prostate involve epigenetic regulation of the cell cycle, apoptosis, cellular antioxidant defenses, and chronic inflammation. Understanding the role of these epigenetic-related mechanisms in humans is an important future area of research.

\section{Cell Cycle and Apoptosis}

Uninhibited cell growth and evasion of apoptosis are classical hallmarks of cancer [33], and SFN has been shown to induce G1/S and G2/M cell cycle arrest and apoptosis specifically in human prostate cancer cells compared to non-cancerous cells $[14,34]$. There is also in vivo evidence of SFN efficacy in prostate cancer prevention. SFN-fed mice showed a reduction in prostate tumor growth that was associated with increased apoptosis and decreased cell proliferation [25]. Furthermore, SFN consumption has been associated with increased expression of $\mathrm{p} 21$, cyclin D, Bax, caspase-3, Bak, and death receptors DR4 and DR5 and decreased expression of Bcl-2 and Bcl$\mathrm{X}_{\mathrm{L}}$ in prostate tissues $[12,13,25,26]$. Similar observations were made when whole-food sources of SFN (broccoli and broccoli sprouts) were consumed $[35,36]$. Some of these effects may be mediated by SFN's ability to inhibit HDACs. For example, mice that consumed $6 \mu \mathrm{mol}$ SFN daily for 10 weeks had decreased levels of HDAC activity, increased acetylated histones $\mathrm{H} 3$ and $\mathrm{H} 4$, and increased expression of $\mathrm{p} 21$ in the prostate [13]. Like p21, cyclin D, Bak, Bax, Bcl-2, Bcl$\mathrm{X}_{\mathrm{L}}$, caspase-3, DR4 and DR5 are often dysregulated in cancer cells through epigenetic modifications, thus SFN could be inducing changes in their expression through epigenetics mechanisms in vivo [37-40]. Recent research in cancer cells supports this idea. For example, in human prostate cancer cells treated with SFN, increased cyclin D2 expression was associated with decreased DNA methylation in its promoter. In these cells, SFN treatment also decreased mRNA and protein levels of DNMTs [19]. 


\section{Antioxidant Defenses}

Cellular antioxidant defenses protect cells against the damaging effects of oxidative stress and inflammation [41, 42]. SFN has been shown to stimulate these defense mechanisms in vivo, often through inducing Nrf2 [43, 44]. Keum et al. [36] reported that dried, ground broccoli sprouts administered in the diet increased Nrf2 and decreased Keap1 protein in mice. These changes were associated with induction of an Nrf2 target gene, heme oxygenase-1 (HO-1). Additionally, increases were observed in the activities of $\mathrm{NAD}(\mathrm{P}) \mathrm{H}$ :quinone oxidoreductase 1 (NQO1), the specific glutathione $S$-transferase (GST) GSTM, and of total GSTs in the prostates of SFN-fed rats [45]. In rat prostates, Liu et al. [46] observed modest decreases in GSTP1 mRNA following consumption of a broccoli powder.

SFN is classically thought to induce Nrf2 by reacting with cysteine residues on Keap1, the cytosolic repressor/chaperone for Nrf2 [47]. Yet, recent evidence suggests that epigenetic mechanisms also contribute to SFN's regulation of Nrf2mediated gene expression. Increased site-specific $\mathrm{CpG}$ methylation within the Nrf2 promoter was associated with reduced Nrf2 expression in mouse prostate tumors [48]. In prostate cancer cells, Zhang et al. [15••] demonstrated that SFN could derepress Nrf2 by reducing $\mathrm{CpG}$ methylation. In these cells, they also observed dose-dependent decreases in expression of DNMT1, DNMT3a, and HDACs 1, 4, 5, and 7 and increased binding of acetylated histone $\mathrm{H} 3$ (Ac-H3) to the Nrf2 promoter following SFN treatment. Epigenetic mechanisms have also been implicated in regulating expression of Keap1 and several GST enzymes [49-52]. There is likely interplay among the genetic and epigenetic mechanisms to elicit the chemopreventive effects of SFN, but more work is needed to understand the precise influence of SFN on its targets in the prostate.

\section{Inflammation}

Inflammation is a major driver of carcinogenesis [33], and epigenetic events play a role in inflammation-mediated cell transformation [53]. Consumption of Brassica vegetables has been shown to reduce markers of systemic oxidative stress and inflammation [54]. A major player that regulates inflammation is the transcription factor, nuclear factor kappa-lightchain enhancer of activated B cells (NF-kB) [53]. NF-kB has increased activity in prostate cancer and regulates the expression of many pro-inflammatory mediators through a welldescribed signaling pathway $[55,56]$. In mice, SFN consumption was shown to reduce NF- $\mathrm{KB}$ activity and the expression of several NF- $\mathrm{kB}$ targets in prostate cancer cell xenografts. These targets included pro-inflammatory mediators interleukins IL-6 and IL-8, hypoxia-inducible factor $1 \alpha$ (HIF-1 $\alpha$ ), and cyclooxygenase-2 (COX-2) [25]. Several of these genes are known to be regulated by various epigenetic mechanisms, so it is possible that epigenetic alterations induced by SFN contributed to these changes in gene expression [39, 57]. For example, in prostate cancer cells, miR-101 inhibits COX-2 post-transcriptional expression [58], and IL- 6 is regulated by the miRNA, let-7, in breast epithelial cells [53]. Additionally, stress-induced increases in IL-6 expression in mouse myoblasts were attenuated following treatment with the HDAC inhibitor, trichostatin A [59]. Importantly, Wong et al. [20] demonstrated that, in prostate cancer cells, $15 \mu \mathrm{M}$ SFN significantly altered the DNA methylation status of the promoters of many genes that regulate inflammation and immune development, including the promoter of IL-6. These data support the possibility that SFN may work through epigenetic mechanisms in prostate tissue to reduce inflammation.

\section{Breast Cancer}

Several epidemiological studies have also indicated that consuming CV may help prevent breast cancer [60-62]. Further evidence of SFN efficacy in breast cancer prevention comes from dietary intervention studies conducted in rats, where consumption of SFN, broccoli, and broccoli sprout extracts was associated with reductions in multiplicity, size, and growth rate of mammary tumors and breast cancer cell xenografts [4, 23•, 63-66]. Similar studies in humans are only starting to emerge, though there is evidence that dietary SFN can reach the breast tissue in humans. Cornblatt et al. [67] demonstrated in healthy, premenopausal women that consuming a broccoli sprout extract beverage containing SFN resulted in measureable levels of SFN metabolites in plasma, urine, and breast tissue within $24 \mathrm{~h}$. This group also detected SFN metabolites in rat mammary tissues following SFN gavage and observed concurrent alterations in antioxidant gene expression. As discussed earlier (SFN and Epigenetics in Prostate and Breast Cancer section), SFN decreased the activity and expression of HDACs, DNMTs, and the HMT, SUV39H1, and altered histone marks, DNA methylation, and miRNA expression in human breast cancer cell lines [16-20, 23•]. Below, we discuss the emerging evidence that these epigenetic mechanisms may mediate certain effects of SFN in the breast, including regulation of antioxidant defenses, cell cycling, apoptosis, and signaling within cancer stem cells (CSC) and tumor microenvironments. Additionally, several SFN targets that are epigenetically regulated in the prostate (e.g., Bcl-2, COX-2) are also altered in breast cancer cells $[68,69]$. Investigations to determine SFN's impact on these targets in breast tissue will provide further information on SFN's role in breast cancer prevention.

\section{Antioxidant Defenses}

SFN may be able to reduce oxidative stress in breast tissue by modulating the expression of antioxidant mediators. Several 
of these proteins are regulated by Nrf2 [47], and SFN has been shown to increase Nrf2 expression and activity in breast cell lines [70]. In rat mammary glands, SFN consumption increased the expression of NQO1 and HO-1, and activity of NQO1, with concurrent increases in the tissue levels of SFN and SFN metabolites [67]. Increased NQO1 and GST activities were also observed in mouse mammary glands following SFN intake [71]. Importantly, Cornblatt et al. [67] demonstrated that NQO1 and HO-1 were detectable in human breast tissues, implicating that these genes could be useful for studying SFN mechanisms in human populations. Evidence from work in SFN-treated, human breast cancer cells suggests that SFN effects on NQO1, HO-1, and GSTs in the breast are mediated in part through epigenetic events [72, 73]. For example, in these cells, SFN exposure increased HO-1 and NQO1 expression, and these changes were associated with increased HAT (p300) recruitment to gene promoters and site-specific increases in H3K9Ac [72].

SFN also increases other antioxidant mediators in breast cells. In cancerous and non-tumorigenic, human breast epithelial cells, SFN exposure increased thioredoxin reductase 1 (TR1), ferritin, light polypeptide (FTL), and proteins involved in glutathione (GSH) metabolism, specifically catalytic and modifier subunits of glutamate-cysteine ligase (GCLC, GCLM), glutathione reductase (GSR), glutaredoxin 1 (GLRX), glutaminase (GLS), gamma-glutamyltransferase 1 (GGT1), and gamma-glutamyltransferase-like activity 4 (GGTLA4) [74, 75]. Epigenetic mechanisms have been implicated in regulating the expression of these genes [76-82]. For example, DNA methylation-dependent regulation of TR1 expression was demonstrated in human breast cancer cells, where a demethylating agent increased TR1 protein levels [78].

\section{Cell Cycle and Apoptosis}

Several studies have demonstrated SFN's ability to disrupt cell growth and proliferation and induce apoptosis in the breast. In mice, SFN injections decreased growth of breast cancer cell xenografts. These xenografts exhibited decreased cell proliferation and Ki-67 staining and increased apoptosis and staining of terminal nucleotidyl transferase-mediated nick end labeling (TUNEL) in a dose-dependent manner with SFN treatment [65]. Stearns et al. [83] reported decreased Ki-67 expression in human breast cancer biopsies following treatment with the pharmacological HDAC inhibitor, Vorinostat, suggesting that epigenetic events play a role in regulating Ki-67. Since SFN can inhibit HDAC activity in breast cancer cells [17], it may work through a similar mechanism to alter expression of Ki-67 in breast tissue.

SFN has been shown to alter the expression of additional cell cycle and apoptotic regulators in breast cancer cell lines. Meeran et al. [18] reported decreased hTERT expression in human breast cancer cells following SFN treatment. In these cells, decreased DNMT expression and $\mathrm{CpG}$ methylation within the binding region of hTERT's repressor, CTCF, occurred along with increased repressor binding. These events were accompanied by decreased HDAC activity, increased expression of RBP2, a HDM that regulates hTERT, and changes in histone acetyl and methyl marks within the hTERT promoter. In a separate study, SFN treatment increased expression of estrogen receptor (ER), epidermal growth factor receptor (EGFR), human epidermal growth factor receptor 2 (HER2), cyclin B1, Fas ligand, and Bcl-2 in human breast cancer cell lines [17]. Decreased HDAC activity was also observed in these cells following SFN treatment, but interestingly, without apparent changes in global histone acetylation. These changes were also associated with G2/M arrest and apoptosis.

SFN likely works through multiple epigenetic mechanisms to elicit changes in gene expression. In fact, both DNA methylation and histone acetylation were shown to be involved in regulating ER expression [84]. There is also evidence that epigenetic regulation contributes to changes in cyclin B1, Bcl-2, and HER-2 expression, but these mechanisms need to be validated in breast tissue [85-87]. Similar to prostate cancer, the chemopreventive effects of SFN may be attributed to a combination of genetic and epigenetic targets.

\section{Cancer Stem Cell and Tumor Microenvironment Signaling}

Dysregulation of cancer stem cell (CSC) signaling can increase risk of tumor development and progression [88]. SFN may be able to alter signaling in breast CSCs and the tumor microenvironment through epigenetic modulation. Li et al. [23•] reported that tumor-suppressive miR-140 was consistently decreased in early-stage and invasive subtypes of breast cancer compared to non-cancerous mammary cells and tissues. In breast CSCs derived from these subtypes, SFN restored expression of miR-140 by decreasing DNA methylation at a specific intronic gene locus. When breast CSCs were injected into mice, tumors resulting from CSCs that had been pre-treated with SFN had higher levels of miR-140, reduced expression of tumor-promoting CSC regulators (SOX9 and ALDH1), and decreased tumor size compared to tumors resulting from non-treated breast $\mathrm{CSCs}\left[23^{\bullet}\right]$. The ability of SFN to regulate CSC signaling in these tumors presents an opportunity for preventing the development of aggressive and therapy-resistant breast cancers.

There is also evidence that SFN interferes with the crosstalk that occurs between adipocytes and mammary stem cells that influences tumor promotion. When SFN was added to cultures containing breast CSCs and adipocytes, CSC migration was markedly reduced. When co-cultured CSCs were pre-treated with SFN and injected into nude mice, resulting tumors were much smaller and stopped growing sooner than tumors arising from untreated, co-cultured CSCs. Similar 
results on tumor growth were found when mice were injected with SFN daily following xenograft implantation [89]. IL-6 mRNA was also lower than in untreated CSCs, suggesting that SFN's interference in the adipocyte-CSC cross-talk was mediated by altering cytokine expression [90]. This finding is highly relevant for cancer chemoprevention strategies, because adipocyte-secreted cytokines have been shown to promote tumor development and migration [91]. Furthermore, there may be an epigenetic interconnection with regard to IL-6 expression, as decreases in IL-6 promoter methylation have been observed in human breast cancer cells due to p53 deficiency [92]. This decrease in IL-6 methylation was associated with increases in IL-6 expression and an epigenetic reprogramming of the cells toward a basal-like/stem cell-like gene expression profile. This area of research warrants additional studies to investigate the mechanisms by which SFN may alter cross-talk between tumor cells and adipocytes in vivo.

\section{Remaining Challenges and Research Needs}

While there is evidence that SFN consumption may be beneficial for breast and prostate cancer chemoprevention, the optimal supplementation form and dosing regimens for SFN in humans still need to be established. This effort will involve clarifying the bioavailability and distribution of SFN and its specific metabolites to human tissues. To date, feeding studies and clinical trials investigating the effects of SFN use a variety of crucifers and extracts to deliver SFN or its precursors, but circumstances that dictate the use of specific sources, forms, or combinations are still emerging. In human feeding studies, consumption of raw or slightly cooked CV resulted in higher levels of SFN in the body as compared to boiled or steamed vegetables $[93,94]$. High cooking temperatures are thought to inactivate the myrosinase enzyme that is needed for deriving SFN from its glucosinolate precursor. It is possible that higher SFN bioavailability confers enhanced chemopreventive activity, substantiating the need for further work in this area as well as efficacy studies in humans. Fresh broccoli sprouts with active myrosinase have been identified as particularly rich dietary sources of SFN, but the high variability in SFN yield among sprout batches create logistical challenges for clinical researchers [95]. Supplemental forms of SFN or its precursor, GFN, have been used to circumvent issues of varying SFN yields from food sources; however, these forms can result in lower SFN absorption than from broccoli sprouts [96]. In an effort to improve SFN absorption from plant extracts, Cramer and Jeffery [97] demonstrated that SFN absorption from a GFN-rich broccoli powder devoid of myrosinase activity was enhanced when co-consumed with fresh broccoli sprouts. Results from another study raised the question of whether or not a combination of GFN and SFN sources can achieve additional benefits than consuming either alone [98]. More research is needed to evaluate and optimize specific formulations, combinations, and dose schedules for SFN delivery in vivo and especially in human subjects.

A better understanding of SFN distribution to target tissues will also help to establish the SFN doses and dosing schedules that achieve effective tissue concentrations. Understanding the distribution of specific metabolites of SFN is important given that the parent compound and metabolites may have differing molecular targets and mechanisms of action. For example, SFN has been implicated as the compound responsible for releasing Nrf2 from Keap1, whereas SFN-cysteine and/or SFN-N'acetylcysteine may be responsible for inhibiting HDACs $[5,99,100]$. SFN metabolites have been observed in multiple animal tissues (i.e., adipose, bladder, brain, breast, colon, duodenum, heart, jejunum, kidney, liver, lung, pancreas, prostate, rectum, and skeletal muscle) [27, 28, 67], but SFN compounds have been detected in humans only in breast tissue [67]. Access to human tissues is limited, generally as clinical biopsies obtained from medical procedures not necessarily linked to predetermined research objectives. This highlights the importance of engaging in research collaborations and optimizing sample preparations to maximize the use of clinical biopsies and animal tissues for research purposes.

Another challenge for clinical researchers is the interindividual variability in SFN metabolism, which has been observed in many controlled human feeding studies. The factors underlying variability in SFN metabolism remain poorly understood. Thus, it is important to identify and characterize key factors that may impact SFN metabolism and distribution to tissues. Such factors include the presence or severity of disease, SFN formulation or diet preparation, tissue type, gut microbiota composition, and genotype of GSTs [101]. Pharmacokinetic studies designed to systematically evaluate the impact of putative factors should consider stratifying subjects prior to randomization to increase statistical power and maximize the value of observations.

Ultimately, establishing key targets of SFN action in humans is critical to determine how SFN can be effectively utilized in chemoprevention strategies. It is worth noting that due to the differences in the genetic background between mice and humans, there may be additional challenges in translating SFN targets discovered in rodent models to the clinic. Nevertheless, once established, tissue-specific targets can be used as biomarkers to evaluate the chemopreventive efficacy of dietary SFN strategies in humans, as well as the impact of SFN metabolism and distribution on chemopreventive outcomes. The use of a single target is likely not sufficient for studying the totality of SFN's chemopreventive effects, especially given that one specific target may not be altered significantly prior to or in the early stages of cancer development, which could limit its use as a biomarker at specific disease stages 
[14]. Additionally, the use of genetic (e.g., Nrf2-regulated genes) and epigenetic mediators (e.g., HDAC, DNMT, ncRNAs) either alone or in combination with proliferation and apoptosis markers could improve understanding of the timing and contributions of various epigenetic mechanisms to specific outcomes. Approaches such as metabolomics may also reveal new mechanisms and novel SFN targets within prostate, mammary, and other target tissues of interest. The outcomes of this research will rely on the ability to understand the physiological relevance of changes in SFN targets observed in human studies; thus, it is critical to quantify the effect sizes for SFN targets that are needed to elicit chemopreventive outcomes.

It is clear that SFN has multiple targets of action in the breast and prostate that may be coordinated by both genetic and epigenetic mechanisms. Future research will need to understand relationships among genetic and epigenetic targets as well as among the bioavailability of active compounds and defined molecular targets in tissues. Emerging bioinformatics technologies can evaluate information on a wide range of SFN targets simultaneously and integrate this information with the presence of SFN metabolites, which will help to clarify SFN mechanisms in vivo. In these evaluations, it will be important to consider the disease context and underlying cellular phenotype, as these factors are likely to impact relationships among SFN compounds, SFN targets, and resulting biological outcomes.

\section{Conclusions}

SFN is a promising dietary chemopreventive agent due to its ability to target multiple pathways involved in carcinogenesis. The ability to alter epigenetic events in the breast and prostate may underlie many of SFN's chemopreventive effects in these tissues. More research is needed to determine the impact of SFN-induced changes in epigenetic mechanisms and their cross-talk during cancer development. To inform chemoprevention strategies for breast and prostate cancers, investigations of SFN's chemopreventive efficacy should focus on tissue-specific effects and work to establish effective doses for different disease stages and human subpopulations.

Acknowledgments We gratefully acknowledge technical assistance from Dr. Fred Stevens of the Linus Pauling Institute and grant funding from the National Institutes of Health (CA090890).

\section{Compliance with Ethics Guidelines}

Conflict of Interest Lauren L. Atwell, Laura M. Beaver, Jackilen Shannon, David E. Williams, Roderick H. Dashwood, and Emily Ho declare that they have no conflict of interest.
Use of Animal or Human Subjects This article does not contain any studies with human or animal subjects performed by any of the authors.

\section{References}

Papers of particular interest, published recently, have been highlighted as:

- Of importance

•- Of major importance

1. Kim MK, Park JHY. Cruciferous vegetable intake and the risk of human cancer: epidemiological evidence. Proc Nutr Soc. 2009;68 (01):103-10.

2. Dinkova-Kostova AT, Zhang Y, Naidu SD, Kostov RV, Pheely A, Calabrese V. Sulfhydryl-reactive phytochemicals as dual activators of transcription factors Nrf2 and HSF1. In: Gang DR, editor. 50 years of phytochemistry research. Switzerland: Springer International Publishing; 2013. p. 95-119.

3. Watson GW, Beaver LM, Williams DE, Dashwood RH, Ho E. Phytochemicals from cruciferous vegetables, epigenetics, and prostate cancer. Am Assoc Pharm Sci J. 2013;15(4):951-61.

4. Zhang Y, Kensler TW, Cho C-G, Posner GH, Talalay P. Anticarcinogenic activities of sulforaphane and structurally related synthetic norbornyl isothiocyanates. Proc Natl Acad Sci U S A. 1994;91(8):3147-50.

5. Myzak MC, Dashwood RH. Chemoprotection by sulforaphane: keep one eye beyond Keap1. Cancer Lett. 2006;233(2):208-18.

6. Vaiopoulos AG, Athanasoula KC, Papavassiliou AG. Epigenetic modifications in colorectal cancer: molecular insights and therapeutic challenges. Biochem Biophys Acta (BBA)-Mol Basis Dis. 2014;1842(7):971-80.

7. Dawson MA, Kouzarides T. Cancer epigenetics: from mechanism to therapy. Cell. 2012;150(6):12-27.

8. Varambally S, Cao Q, Mani R-S, Shankar S, Wang X, Ateeq B, et al. Genomic loss of microRNA-101 leads to overexpression of histone methyltransferase EZH2 in cancer. Science. 2008;322 (5908):1695-9.

9. Perry AS, Watson WG, Lawler M, Hollywood D. The epigenome as a therapeutic target in prostate cancer. Nat Rev Urol. 2010;7 (12):668-80.

10. Schrump DS. Cytotoxicity mediated by histone deacetylase inhibitors in cancer cells: mechanisms and potential clinical implications. Clin Cancer Res. 2009;15(12):3947-57.

11. Clarke JD, Dashwood RH, Ho E. Multi-targeted prevention of cancer by sulforaphane. Cancer Lett. 2008;269(2):291-304.

12. Myzak MC, Tong P, Dashwood WM, Dashwood RH, Ho E. Sulforaphane retards the growth of human PC-3 xenografts and inhibits HDAC activity in human subjects. Exp Biol Med. 2007;232(2):227-34.

13. Myzak MC, Dashwood WM, Orner GA, Ho E, Dashwood RH. Sulforaphane inhibits histone deacetylase in vivo and suppresses tumorigenesis in $A p c^{\mathrm{min}}$ mice. FASEB J. 2006;20(3):506-8.

14. Clarke JD, Hsu A, Yu Z, Dashwood RH, Ho E. Differential effects of sulforaphane on histone deacetylases, cell cycle arrest and apoptosis in normal prostate cells versus hyperplastic and cancerous prostate cells. Mol Nutr Food Res. 2011;55(7):999-1009.

15.• Zhang C, Su Z-Y, Khor TO, Shu L, Kong A-NT. Sulforaphane enhances Nrf2 expression in prostate cancer TRAMP C1 cells through epigenetic regulation. Biochem Pharmacol. 2013;85(9): 1398-404. This key study establishes the possible interplay between classic genetic and epigenetic mechanisms of sulforaphane. 
These mechanisms act in concert to increase the expression of Nrf2 and promote chemoprevention.

16. Meeran SM, Patel SN, Li Y, Shukla S, Tollefsbol TO. Bioactive dietary supplements reactivate ER expression in ER-negative breast cancer cells by active chromatin modifications. PLoS One. 2012;7(5):e37748.

17. Pledgie-Tracy A, Sobolewski MD, Davidson NE. Sulforaphane induces cell type-specific apoptosis in human breast cancer cell lines. Mol Cancer Ther. 2007;6(3):1013-21.

18. Meeran SM, Patel S, Tollefsbol TO. Sulforaphane causes epigenetic repression of hTERT expression in human breast cancer cell lines. PLoS One. 2010;5(7):e11457.

19. Hsu A, Wong CP, Yu Z, Williams DE, Dashwood RH, Ho E. Promoter de-methylation of cyclin D2 by sulforaphane in prostate cancer cells. Clin Epigenetics. 2011;3(1):1-9.

20. Wong CP, Hsu A, Buchanan A, Palomera-Sanchez Z, Beaver LM, Houseman EA, et al. Effects of sulforaphane and 3,3'diindolylmethane on genome-wide promoter methylation in normal prostate epithelial cells and prostate cancer cells. PLoS One. 2014;9(1):e86787.

21. Maia BM, Rocha RM, Calin GA. Clinical significance of the interaction between non-coding RNAs and the epigenetics machinery: challenges and opportunities in oncology. Epigenetics. 2014;9(1):75-80.

22. Parasramka MA, Ho E, Williams DE, Dashwood RH. MicroRNAs, diet, and cancer: new mechanistic insights on the epigenetic actions of phytochemicals. Mol Carcinog. 2012;51(3): 213-30.

23. Li Q, Yao Y, Eades G, Zhang Y, Zhou Q. Downregulation of miR140 promotes cancer stem cell formation in basal-like early stage breast cancer. Oncogene. 2014;33(20):2589-600. This is one of the first key papers that established the effect of sulforaphane treatment on miRNA signaling in cancer stem cells and shows that it may play an important role in suppressing cancer formation.

24. Suganuma T, Workman JL. Signals and combinatorial functions of histone modifications. Annu Rev Biochem. 2011;80:473-99.

25. Shankar S, Ganapathy S, Srivastava RK. Sulforaphane enhances the therapeutic potential of TRAIL in prostate cancer orthotopic model through regulation of apoptosis, metastasis, and angiogenesis. Clin Cancer Res. 2008;14(21):6855-66.

26. Singh AV, Xiao D, Lew KL, Dhir R, Singh SV. Sulforaphane induces caspase-mediated apoptosis in cultured PC-3 human prostate cancer cells and retards growth of PC-3 xenografts in vivo. Carcinogenesis. 2004;25(1):83-90.

27.• Veeranki OL, Bhattacharya A, Marshall JR, Zhang Y. Organspecific exposure and response to sulforaphane, a key chemopreventive ingredient in broccoli: implications for cancer prevention. Br J Nutr. 2012;1(1):1-8. This is one of the first studies to establish tissue-specific targets of sulforaphane in vivo by co-examining the distribution of sulforaphane and Phase 2 enzyme responses (GST and NQO1) in multiple tissues in a rat model.

28. Clarke JD, Hsu A, Williams DE, Dashwood RH, Stevens JF, Yamamoto M, et al. Metabolism and tissue distribution of sulforaphane in Nrf2 knockout and wild-type mice. Pharm Res. 2011;28(12):3171-9.

29. Liu B, Mao Q, Cao M, Xie L. Cruciferous vegetables intake and risk of prostate cancer: a meta-analysis. Int J Urol. 2012;19(2): 134-41.

30. Richman EL, Carroll PR, Chan JM. Vegetable and fruit intake after diagnosis and risk of prostate cancer progression. Int $\mathrm{J}$ Cancer. 2012;131(1):201-10.

31. Alumkal JJ, Slottke R, Mori M, Schwartzman J, Graff JN, Beer $\mathrm{TM}$, et al. Sulforaphane treatment in men with recurrent prostate cancer. 2013 ASCO Annual Meeting Abstracts. J Clin Oncol. 2013;31(15):5017
32. Traka M, Gasper AV, Melchini A, Bacon JR, Needs PW, Frost V, et al. Broccoli consumption interacts with GSTM1 to perturb oncogenic signaling pathways in the prostate. PLoS One. 2008;3(7): e2568.

33. Hanahan D, Weinberg RA. Hallmarks of cancer: the next generation. Cell. 2011;144(5):646-74.

34. Beaver LM, Williams DE, Dashwood RH, Ho E. Chemoprevention of prostate cancer with cruciferous vegetables: role of epigenetics. In: Shankar S, Srivastava RK, editors. Nutrition, diet and cancer. Netherlands: Springer Science+ Business Media B.V; 2012. p. 49-81.

35. Canene-Adams K, Lindshield BL, Wang S, Jeffery EH, Clinton SK, Erdman JWJ. Combinations of tomato and broccoli enhance antitumor activity in Dunning R3327-H prostate adenocarcinomas. Cancer Res. 2007;67(2):836-43.

36. Keum YS, Khor TO, Lin W, Shen G, Kwon KH, Barve A, et al. Pharmacokinetics and pharmacodynamics of broccoli sprouts on the suppression of prostate cancer in transgenic adenocarcinoma of mouse prostate (TRAMP) mice: implication of induction of Nrf2, HO-1 and apoptosis and the suppression of Akt-dependent kinase pathway. Pharm Res. 2009;26(10):2324-31.

37. Sanders YY, Hagood JS, Liu H, Zhang W, Ambalavanan N, Thannickal VJ. Histone deacetylase inhibition promotes fibroblast apoptosis and ameliorates pulmonary fibrosis in mice. Eur Respir J. 2014;43(5):1448-58.

38. Carvalho JR, Filipe L, Costa VL, Ribeiro FR, Martins AT, Teixeira MR, et al. Detailed analysis of expression and promoter methylation status of apoptosis-related genes in prostate cancer. Apoptosis. 2010;15(8):956-65.

39. Glozak MA, Seto E. Histone deacetylases and cancer. Oncogene. 2007;26(37):5420-32.

40. Nakata S, Yoshida T, Horinaka M, Shiraishi T, Wakada M, Sakai T. Histone deacetylase inhibitors upregulate death receptor 5/TRAIL-R2 and sensitize apoptosis induced by TRAIL/APO2L in human malignant tumor cells. Oncogene. 2004;23(37):626171.

41. Khor TO, Huang M-T, Kwon KH, Chan JY, Reddy BS, Kong AN. Nrf2-deficient mice have an increased susceptibility to dextran sulfate sodium-induced colitis. Cancer Res. 2006;66(24): $11580-4$.

42. Khor TO, Huang M-T, Prawan A, Liu Y, Hao X, Yu S, et al. Increased susceptibility of Nrf2 knockout mice to colitisassociated colorectal cancer. Cancer Prev Res. 2008;1(3):187-91.

43. Hu R, Xu C, Shen G, Jain MR, Khor TO, Gopalkrishnan A, et al. Gene expression profiles induced by cancer chemopreventive isothiocyanate sulforaphane in the liver of $\mathrm{C} 57 \mathrm{BL} / 6 \mathrm{~J}$ mice and C57BL/6J/Nrf2 (-/-) mice. Cancer Lett. 2006;243(2):170-92.

44. Fahey JW, Haristoy X, Dolan PM, Kensler TW, Scholtus I, Stephenson KK, et al. Sulforaphane inhibits extracellular, intracellular, and antibiotic-resistant strains of Helicobacter pylori and prevents benzo[a]pyrene-induced stomach tumors. Proc Natl Acad Sci U S A. 2002;99(11):7610-5.

45. Jones SB, Brooks JD. Modest induction of phase 2 enzyme activity in the F-344 rat prostate. Biomed Cent Cancer. 2006;6(1):62 70.

46. Liu AG, Volker SE, Jeffery EH, Erdman Jr JW. Feeding tomato and broccoli powder enriched with bioactives improves bioactivity markers in rats. J Agric Food Chem. 2009;57(16):7304-10.

47. Kwak MK, Kensler TW. Targeting Nrf2 signaling for cancer chemoprevention. Toxicol Appl Pharmacol. 2010;244(1):66-76.

48. Yu S, Khor TO, Cheung K-L, Li W, Wu T-Y, Huang Y, et al. Nrf2 expression is regulated by epigenetic mechanisms in prostate cancer of TRAMP mice. PLoS One. 2010;5(1):e8579.

49. Cheng X, Ku C-H, Siow RC. Regulation of the Nrf2 antioxidant pathway by microRNAs: new players in micromanaging redox homeostasis. Free Radic Biol Med. 2013;64(9):4-11. 
50. Wang LG, Beklemisheva A, Liu X-M, Ferrari AC, Feng J, Chiao J-W. Dual action on promoter demethylation and chromatin by an isothiocyanate restored GSTP1 silenced in prostate cancer. Mol Carcinog. 2007;46(1):24-31.

51. Pandey M, Shukla S, Gupta S. Promoter demethylation and chromatin remodeling by green tea polyphenols leads to re-expression of GSTP1 in human prostate cancer cells. Int J Cancer. 2010;126 (11):2520-33.

52. Cui JY, Choudhuri S, Knight TR, Klaassen CD. Genetic and epigenetic regulation and expression signatures of glutathione $\mathrm{S}$ transferases in developing mouse liver. Toxicol Sci. 2010;116(1): $32-43$.

53. Iliopoulos D, Hirsch HA, Struhl K. An epigenetic switch involving NF- $\mathrm{KB}$, Lin28, Let-7 microRNA, and IL6 links inflammation to cell transformation. Cell. 2009;139(4):693-706.

54. Fowke JH, Morrow JD, Motley S, Bostick RM, Ness RM. Brassica vegetable consumption reduces urinary F2-isoprostane levels independent of micronutrient intake. Carcinogenesis. 2006;27(10):2096-102.

55. Gasparian AV, Yao YJ, Kowalczyk D, Lyakh LA, Karseladze A, Slaga TJ, et al. The role of IKK in constitutive activation of NF-KB transcription factor in prostate carcinoma cells. J Cell Sci. 2002;115(1):141-51.

56. Suh J, Payvandi F, Edelstein LC, Amenta PS, Zong W-X, Gélinas $\mathrm{C}$, et al. Mechanisms of constitutive NF- $\mathrm{KB}$ activation in human prostate cancer cells. Prostate. 2002;52(3):183-200.

57. Hoffmann E, Dittrich-Breiholz O, Holtmann H, Kracht M. Multiple control of interleukin- 8 gene expression. J Leukoc Biol. 2002;72(5):847-55.

58. Hao Y, Gu X, Zhao Y, Greene S, Sha W, Smoot DT, et al. Enforced expression of miR-101 inhibits prostate cancer cell growth by modulating the COX-2 pathway in vivo. Cancer Prev Res. 2011;4(7):1073-83.

59. Frost RA, Nystrom GJ, Lang CH. Epinephrine stimulates IL-6 expression in skeletal muscle and $\mathrm{C}_{2} \mathrm{C}_{12}$ myoblasts: role of c-Jun $\mathrm{NH}_{2}$-terminal kinase and histone deacetylase activity. Endocrinol Metab. 2004;286(5):E809-17.

60. Terry P, Wolk A, Piersson I, Mangnusson C. Brassica vegetables and breast cancer risk. J Am Med Assoc. 2001;285(23):2975-7.

61. Fowke JH, Chung FL, Jin F, Qi D, Cai Q, Conaway C, et al. Urinary isothiocyanate levels, Brassica, and human breast cancer. Cancer Res. 2003;63(14):3980-6.

62. Ambrosone CB, McCann SE, Freudenheim JL, Marshall JR, Zhang Y, Shields PG. Breast cancer risk in premenopausal women is inversely associated with consumption of broccoli, a source of isothiocyanates, but is not modified by GST genotype. J Nutr. 2004;134(5):1134-8.

63. Finley JW, Ip C, Lisk DJ, Davis CD, Hintze KJ, Whanger PD. Cancer-protective properties of high-selenium broccoli. J Agric Food Chem. 2001;49(5):2679-83.

64. Fahey JW, Zhang Y, Talalay P. Broccoli sprouts: an exceptionally rich source of inducers of enzymes that protect against chemical carcinogens. Proc Natl Acad Sci U S A. 1997;94(19):10367-72.

65. Kanematsu S, Yoshizawa K, Uehara N, Miki H, Sasaki T, Kuro M, et al. Sulforaphane inhibits the growth of KPL-1 human breast cancer cells in vitro and suppresses the growth and metastasis of orthotopically transplanted KPL-1 cells in female athymic mice. Oncol Rep. 2011;26(3):603-8.

66. Jackson SJ, Singletary KW. Sulforaphane: a naturally occurring mammary carcinoma mitotic inhibitor, which disrupts tubulin polymerization. Carcinogenesis. 2004;25(2):219-27.

67. Cornblatt BS, Ye L, Dinkova-Kostova AT, Erb M, Fahey JW, Singh NK, et al. Preclinical and clinical evaluation of sulforaphane for chemoprevention in the breast. Carcinogenesis. 2007;28(7): 1485-90.
68. Hussain A, Mohsin J, Prabhu SA, Begum S, Nusri QE-A, Harish $\mathrm{G}$, et al. Sulforaphane inhibits growth of human breast cancer cells and augments the therapeutic index of the chemotherapeutic drug, gemcitabine. Asian Pac J Cancer Prev. 2013;14(10):5855-60.

69. Ristamäki A, Sivula A, Lundin J, Lundin M, Salminen T, Haglund $\mathrm{C}$, et al. Prognostic significance of elevated cyclooxygenase-2 expression in breast cancer. Cancer Res. 2002;62(3):632-5.

70. Kang HJ, Hong YB, Kim HJ, Wang A, Bae I. Bioactive food components prevent carcinogenic stress via Nrf2 activation in BRCA1 deficient breast epithelial cells. Toxicol Lett. 2012;209(2): 154-60.

71. Gerhäuser C, You M, Liu J, Moriarty RM, Hawthorne M, Mehta $\mathrm{RG}$, et al. Cancer chemopreventive potential of sulforamate, a novel analogue of sulforaphane that induces phase 2 drugmetabolizing enzymes. Cancer Res. 1997;57(2):272-8.

72. Lo R, Matthews J. The aryl hydrocarbon receptor and estrogen receptor alpha differentially modulate nuclear factor erythroid-2related factor 2 transactivation in MCF-7 breast cancer cells. Toxicol Appl Pharmacol. 2013;270(2):139-48.

73. Lee JS. GSTP1 promoter hypermethylation is an early event in breast carcinogenesis. Virchows Arch. 2007;450(6):637-42.

74. Wang W, Wang S, Howie AF, Beckett GJ, Mithen R, Bao Y. Sulforaphane, erucin, and iberin up-regulate thioredoxin reductase 1 expression in human MCF-7 cells. J Agric Food Chem. 2005;53 (5):1417-21.

75. Agyeman AS, Chaerkady R, Shaw PG, Davidson NE, Visvanathan K, Pandey A, et al. Transcriptomic and proteomic profiling of KEAP1 disrupted and sulforaphane-treated human breast epithelial cells reveals common expression profiles. Breast Cancer Res Treat. 2012;132(1):175-87.

76. Chiba T, Yokosuka O, Arai M, Tada M, Fukai K, Imazeki F, et al. Identification of genes up-regulated by histone deacetylase inhibition with cDNA microarray and exploration of epigenetic alterations on hepatoma cells. J Hepatol. 2004;41(3):436- 45.

77. Chekhun VF, Lukyanova NY, Burlaka AP, Bezdenezhnykh NA, Shpyleva SI, Tryndyak VP, et al. Iron metabolism disturbances in the MCF-7 human breast cancer cells with acquired resistance to doxorubicin and cisplatin. Int J Oncol. 2013;43(5):1481-6.

78. Rivenbark AG, Jones WD, Risher JD, Coleman WB. DNA methylation-dependent epigenetic regulation of gene expression in MCF-7 breast cancer cells. Epigenetics. 2006;1(1):32-44.

79. Higgs G, Slack F. The multiple roles of microRNA-155 in oncogenesis. J Clin Bioinforma. 2013;3(1):17-24.

80. Kim JH, Kang H-S, Kim TW, Kim SJ. Differential methylation hybridization profiling identifies involvement of STAT1-mediated pathways in breast cancer. Int J Oncol. 2011;39(4):955-63.

81. Chung SA, French J, Taylor KE, Elboudwarej E, Quach HL, Barcellos LF, et al. Differential DNA methylation associated with anti-dsDNA autoantibody production in systemic lupus erythematosus. Arthritis Res Ther. 2012;14(S3):A6.

82. Yakabe S, Soejima H, Yatsuki H, Tominaga H, Zhao W, Higashimoto $\mathrm{K}$, et al. MeCP2 knockdown reveals DNA methylation-independent gene repression of target genes in living cells and a bias in the cellular location of target gene products. Genes Genet Syst. 2008;83(2):199-208.

83. Stearns V, Jacobs LK, Fackler M, Tsangaris TN, Rudek MA, Higgins M, et al. Biomarker modulation following short-term Vorinostat in women with newly diagnosed primary breast cancer. Clin Cancer Res. 2013;19(14):4008-16.

84. Yang X, Phillips DL, Ferguson AT, Nelson WG, Herman JG, Davidson NE. Synergistic activation of functional estrogen receptor (ER)- $\alpha$ by DNA methyltransferases and histone deacetylase inhibition in human ER- $\alpha$-negative breast cancer cells. Cancer Res. 2001;61(19):7025-9. 
85. Huang V, Place RF, Portnoy V, Wang J, Qi Z, Jia Z, et al. Upregulation of cyclin B1 by miRNA and its implications in cancer. Nucleic Acids Res. 2011;40(4):1695-707.

86. Yang J, Cao Y, Sun J, Zhang Y. Curcumin reduces the expression of Bcl-2 by upregulating miR-15a and miR-16 in MCF-7 cells. Med Oncol. 2010;27(4):1114-8.

87. Fuino L, Bali P, Wittmann S, Donapaty S, Guo F, Yamaguchi H, et al. Histone deacetylase inhibitor LAQ824 down-regulates Her-2 and sensitizes human breast cancer cells to trastuzumab, taxotere, gemcitabine, and epothilone B. Mol Cancer Ther. 2003;2(10): 971-84.

88. Visvader JE, Lindeman GJ. Cancer stem cells in solid tumours: accumulating evidence and unresolved questions. Nat Rev Cancer. 2008;8(10):755-68.

89. Li Y, Zhang T, Korkaya H, Liu S, Lee H-F, Newman B, et al. Sulforaphane, a dietary component of broccoli/broccoli sprouts, inhibits breast cancer stem cells. Clin Cancer Res. 2010;16(9): 2580-90.

90. Li Q, Xia J, Yao Y, Gong D-W, Shi H, Zhou Q. Sulforaphane inhibits mammary adipogenesis by targeting adipose mesenchymal stem cells. Breast Cancer Res Treat. 2013;141(2):317-24.

91. Iyengar P, Combs TP, Shah SJ, Gouon-Evans V, Pollard JW, Albanese $\mathrm{C}$, et al. Adipocyte-secreted factors synergistically promote mammary tumorigenesis through induction of anti-apoptotic transcriptional programs and proto-oncogene stabilization. Oncogene. 2003;22(41):6408-23.

92. D'Anello L, Sansone P, Storci G, Mitrugno V, D'Uva G, Chieco P, et al. Epigenetic control of the basal-like gene expression profile via Interleukin-6 in breast cancer cells. Mol Cancer. 2010;9:300.

93. Vermeulen M, Klopping-Ketelaars IWAA, Van den Berg R, Vaes WH. Bioavailability and kinetics of sulforaphane in humans after consumption of cooked versus raw broccoli. J Agric Food Chem. 2008;56(22):10505-9.
94. Conaway CC, Getahun SM, Liebes LL, Pusateri DJ, Topham DKW, Botero-Omary M, et al. Disposition of glucosinolates and sulforaphane in humans after ingestion of steamed and fresh broccoli. Nutr Cancer. 2000;38(2):168-78.

95. Fahey JW, Wehage SL, Holtzclaw WD, Kensler TW, Egner PA, Shapiro TA, et al. Protection of humans by plant glucosinolates: efficiency of conversion of glucosinolates to isothiocyanates by the gastrointestinal microflora. Cancer Prev Res. 2012;5(4):60311.

96. Clarke JD, Hsu A, Riedl K, Bella D, Schwartz SJ, Stevens JF, et al. Bioavailability and inter-conversion of sulforaphane and erucin in human subjects consuming broccoli sprouts or broccoli supplement in a cross-over study design. Pharmacol Res. 2011;64(5): 456-63.

97. Cramer JM, Jeffery EH. Sulforaphane absorption and excretion following ingestion of a semi-purified broccoli powder rich in glucoraphanin and broccoli sprouts in healthy men. Nutr Cancer. 2011;63(2):196-201.

98. Egner PA, Chen JG, Wang JB, Wu Y, Sun Y, Lu JH, et al. Bioavailability of sulforaphane from two broccoli sprout beverages: results of a short-term cross-over clinical trial in Qidong, China. Cancer Prev Res. 2011;4(3):384-95.

99. Myzak MC, Karplus PA, Chung FL, Dashwood RH. A novel mechanism of chemoprotection by sulforaphane. Cancer Res. 2004;64(16):5767-74.

100. Rajendran P, Kidane AI, Yu T-W, Dashwood W-M, Bisson WH, Löhr C, et al. HDAC turnover, CtIP acetylation and dysregulated DNA damage signaling in colon cancer cells treated with sulforaphane and related isothiocyanates. Epigenetics. 2013;8(6):61223.

101. Lampe JW, Chang J-L. Interindividual differences in phytochemical metabolism and disposition. Semin Cancer Biol. 2007;17(5): $347-53$. 